\title{
Medida da cor em solos do Rio Grande do Sul com a carta de Munsell e por colorimetria
}

\author{
Color measurement in soils from Rio Grande do Sul State with Munsell charts and by colorimetry
}

\author{
Márcio Ramos Botelho ${ }^{1}$ Ricardo Simão Diniz Dalmolin² ${ }^{2}$ abrício de Araújo Pedron ${ }^{2}$ \\ Antonio Carlos de Azevedo ${ }^{2}$ Rodrigo Borkowski Rodrigues ${ }^{3}$ Pablo Miguel $^{3}$
}

\section{RESUMO}

A cor do solo é uma característica facilmente determinada através da comparação visual com a carta de Munsell e está relacionada com a presença de óxidos de ferro e matéria orgânica no solo. A obtenção da cor por instrumentos via sensoriamento remoto, como colorímetros, resulta em maior precisão por apresentar condições controladas e não subjetivas. Os objetivos deste trabalho foram comparar a cor de diferentes solos do Rio Grande do Sul determinadas pela carta de Munsell e por colorimetria e correlacioná-las com os atributos do solo. A precisão dos observadores na determinação da cor através da carta de Munsell para uma mesma amostra apresentou menor variabilidade para o componente matiz e maior para o croma. Os coeficientes de correlação mostraram que há coerência entre as medidas de cor realizadas por diferentes observadores e as medidas pelo colorímetro, o qual mostrou ser eficiente na quantificação da cor, eliminando possíveis erros psicofísicos atribuídos ao método visual. Os atributos dos solos analisados apresentaram boas correlações com os componentes da cor obtidos por colorimetria, mostrando ser esta uma técnica eficiente e promissora para obter informações do solo de maneira simples e de baixo custo.

Palavras-chave: pedologia, morfologia do solo, gênese do solo, sensoriamento remoto.

\section{ABSTRACT}

The soil color is easily determined through the visual comparison with the Munsell charts and relates with the presence of iron oxides and organic matter in the soil. The color measurement by instruments through remote sensing has greater precision due to controlled, non-subjective conditions. The aims of this research were to compare the color of different soils from Rio Grande do Sul State Brazil measured by the
Munsell charts and colorimetry and to correlate the color with the soil characteristics. The smallest variability in determining color through Munsell chart was observed in the hue component and the largest in the chrome. The correlation coefficients showed that there is coherence among the color measurements using Munsell chart and the colorimeter. The colorimeter was efficient to quantify color, eliminating psicophysics errors typical of the visual method. The characteristics of the soils analyzed presented high correlations with the components of the color obtained by colorimetry, inidicating this technique as efficient and promising to obtain information of the soil in a simple way with low cost.

Key words: pedology, soil morphology, soil genesis, remote sensing.

\section{INTRODUÇÃO}

A cor é uma das mais importantes características do solo, sendo considerada na sua identificação e na sua descrição no campo, além de ser um atributo diferencial para muitas classes de solos nos sistemas de classificação. A matéria orgânica e os óxidos de ferro são os principais agentes responsáveis pela cor dos solos. Este fato justifica o empenho de inúmeros pesquisadores na tentativa de determinar o atributo cor com maior exatidão, estabelecendo relações quantitativas entre esta e os constituintes do solo (SCHULZE et al., 1993; BARRÓN et al., 2000; CAMPOS et al., 2003).

\footnotetext{
${ }^{1}$ Programa de Pós-graduação em Ciência do Solo (PPGCS), Universidade Federal de Santa Maria (UFSM), Santa Maria, Rio Grande do Sul (RS), Brasil.

${ }^{2}$ Departamento de Solos, Campus UFSM, 97105-900, Santa Maria, RS, Brasil. E-mail: dalmolinomiln@ccr.ufsm.br. Autor para correspondência.

${ }^{3}$ Curso de Agronomia, UFSM, Santa Maria, RS, Brasil.
} 
A matéria orgânica confere cores escuras aos horizontes superficiais e em alguns horizontes subsuperficiais (iluviação). Cores vermelhas, amarelas e brunadas são atribuídas à presença de óxidos de ferro, enquanto a presença de cores acinzentadas está relacionada aos ambientes de redução e remoção dos óxidos de ferro, em condições hidromórficas. Cores avermelhadas são atribuídas à presença de hematita e índices de avermelhamento são propostos para quantificar este óxido de ferro em solos (BARRON \& TORRENT, 1986). Solos ricos em quartzo e pobres em matéria orgânica e óxidos de ferro apresentam cores claras e esbranquiçadas (AZEVEDO \& DALMOLIN, 2004).

A cor do solo é determinada a campo pela comparação visual de amostras secas e úmidas utilizando-se a carta de Munsell para solos, observandose o matiz (comprimento de onda da luz), o valor (brilho ou tonalidade) e o croma (intensidade ou pureza da cor em relação ao cinza). Este sistema está baseado na percepção visual, sendo utilizado mundialmente pelos pedólogos devido a sua fácil e rápida aplicação em trabalhos de campo. Entretanto, métodos de percepção visual apresentam subjetividade. Os fatores de maior efeito na subjetividade da interpretação da cor pelo olho humano são as características da luz incidente sobre o solo, características da superfície do solo e a qualidade da resposta espectral do olho humano, uma vez que não são fatores controlados (MELVILLE \& ATKINSON, 1985; POST et al., 1993). A obtenção da cor com instrumentos de medida via sensoriamento remoto (colorímetros, espectrofotômetros e espectrorradiômetros) resultam em maior precisão por se darem em condições controladas e não subjetivas.

O colorímetro possui um sensor que quantifica a energia refletida na região do espectro visível, resultante da interação da luz com o solo, utilizando os valores triestímulos básicos propostos pela Commision Internationale L’Eclarirage (CIE, 1931). O sistema CIE L*a*b* estabelece coordenadas uniformes no espaço tridimensional de cor, sendo que "L” é a luminosidade e “a” e "b” contêm as informações de croma, onde "a” corresponde a um eixo que varia do vermelho ao verde e "b” é um eixo que varia do amarelo ao azul (HUNTERLAB, 1996; MINOLTA, 1998).

Vários fatores influenciam a obtenção dos dados, sendo que as características do meio iluminante em que as amostras são visualizadas podem influenciar na aparência da cor (HUNTERLAB, 1996). A umidade da amostra também pode influenciar, pois há aumento na absorção da radiação eletromagnética devido à presença de água, sendo a coloração do solo mais escura quanto maior a umidade (STONER et al., 1991).
Para minimizar as alterações de cor e os efeitos de sombra provocado pelos agregados de solos, as amostras devem ser pulverizadas e tamizadas (BARRÓN et al., 2000; SCHEINOST \& SCHWERTMANN, 1999).

Portanto, considerando que a cor do solo é usualmente determinada por comparação visual e depende da percepção do observador, os objetivos deste trabalho foram determiná-la comparativamente pelo método da carta de Munsell e pelo colorímetro, avaliar a precisão dos observadores na determinação da cor do solo e correlacionar as medidas da cor realizadas por colorimetria com alguns atributos de diferentes solos do Rio Grande do Sul (RS).

\section{MATERIAL E MÉTODOS}

Os solos foram coletados em diferentes regiões fisiográficas do RS: Planalto Médio, Campos de Cima da Serra, Escudo Cristalino, Depressão Central e Campanha, sendo classificados, conforme o Sistema Brasileiro de Classificação de Solos (EMBRAPA, 1999), em: Alissolo Hipocrômico argilúvico típico (APt), Latossolo Bruno alumínico típico (LBa), Latossolo Vermelho distrófico típico (LVd1 e LVd2), Latossolo Vermelho distroférrico típico (LVdf1 e LVdf2), Argissolo Vermelho distrófico latossólico (PVd1), Argissolo Vermelho distrófico típico (PVd2), Argissolo Vermelho distrófico arênico (PVd3), Planossolo Hidromórfico eutrófico arênico (SGe), Planossolo Háplico eutrófico típico (SXe) e Vertissolo Ebânico órtico típico (VEo).

Foram coletadas amostras nas profundidades de $0-20$ e $20-40 \mathrm{~cm}$, as quais foram secadas ao ar, destorroadas e passadas por uma peneira com malha de $2 \mathrm{~mm}$, obtendo-se a terra fina seca ao ar (TFSA). A composição granulométrica foi determinada pelo método da pipeta, conforme EMBRAPA(1997). A determinação dos teores de carbono foi realizada em bloco digestor, conforme YEOMANS \& BREMNER (1988); NELSON \& SOMMERS (1996). A extração de ferro referente aos óxidos de ferro pedogênicos (Fed) foi realizada através da extração com DCB em temperatura ambiente [E-S1](HOLMGREN, 1967) e o teor de óxidos de ferro com baixa cristalinidade (Feo) foi obtido por extração com oxalato de amônio (SCHWERTMANN, 1964).

Os índices de vermelho foram calculados através da expressão IV = [(10-h).c $] / v$, proposta por HURST (1977) e modificada por TORRENT et al. (1980), onde "c" e "v" são os valores numéricos do croma e do valor, respectivamente, "h” é o número que precede o YR no matiz. Para o matiz 10R, o valor do "h” é igual a zero; e para o matiz 10YR, igual a 10. As amostras de solo (TFSA) foram pulverizadas em gral de ágata e 
passadas por uma peneira de malha de $0,250 \mathrm{~mm}$, conforme SCHEINOST \& SCHWERTAMNN (1999), visando à homogeneização da cor da amostra e à obtenção da cor seca triturada, sendo as amostras acondicionadas em placas de petri com diâmetro de 14 $\mathrm{cm}$ e com $2 \mathrm{~cm}$ de altura.

Foram selecionados oito observadores para determinação da cor seca triturada por comparação visual sob fonte de iluminação padronizada. Posteriormente, foi realizada medida da cor com um colorímetro MINOLTA CR-310, que opera na faixa espectral do visível, utilizando o sistema $\mathrm{L}^{*} \mathrm{a} * \mathrm{~b} *$, com fator iluminante D65 e ângulo observador de $2^{\circ}$. Como branco padrão, foi utilizado uma placa de $\mathrm{BaSO}_{4}$. Para converter os valores $\mathrm{L}^{*} \mathrm{a} * \mathrm{~b} *$ para a escala de cores de Munsell, utilizou-se o software Munsell Conversionversão 6.41.

Para análise estatística, foi utilizado o software Statistica, versão 6 (STAT SOFT, Inc., 2001). O componente matiz foi transformado em dado ordinal em uma escala que varia do vermelho para o amarelo, estabelecendo-se a seguinte ordem: $10 \mathrm{R}=1 ; 2,5 \mathrm{YR}=$ $2 ; 5 \mathrm{YR}=3 ; 7,5 \mathrm{YR}=4 ; 10 \mathrm{YR}=5 ; 2,5 \mathrm{Y}=6 \mathrm{e} 5 \mathrm{Y}=7$ (POST et al., 1993). Para valor e croma, não foi necessário fazer transformações. Foram efetuadas correlações entre as medidas de cor feitas por comparação visual e por colorimetria e utilizadas medidas de variabilidade para avaliar as divergências na determinação da cor pelos observadores, considerando a mesma amostra de solo, tais como: amplitude da variação, variância, desvio padrão e coeficiente de variação. Os atributos dos solos e os componentes da cor, determinados pelo colorímetro, foram correlacionados, obtendo-se coeficientes de correlação e equações de regressão lineares, com significância a 5\% $(\mathrm{P}<0,05)$ através do aplicativo estatístico citado.

\section{RESULTADOS E DISCUSSÃO}

A composição granulométrica, os teores de matéria orgânica, os óxidos de ferro e os índices de vermelho dos solos estudados são apresentadas na tabela 1. A granulometria dos solos é influenciada pelo material de origem destes e, juntamente com a composição mineralógica e o teor de matéria orgânica, também está relacionada com a cor do solo. Os solos

Tabela 1 - Granulometria, matéria orgânica, ferro extraído com solução DCB (Fed) e com solução de oxalato de amônio (Feo), relação Feo/Fed e índices de vermelho (IV) para os diferentes solos analisados.

\begin{tabular}{|c|c|c|c|c|c|c|c|c|c|}
\hline \multirow{2}{*}{ Solo } & \multirow{2}{*}{ Prof. (cm) } & Areia & Silte & Argila & MO & Fed & Feo & Feo/Fed & \multirow{2}{*}{ IV } \\
\hline & & \multicolumn{7}{|c|}{$\mathrm{g} \mathrm{kg}^{-1}$} & \\
\hline \multirow{2}{*}{ APt } & $0-20$ & 530 & 372 & 98 & 17 & 4 & 1,7 & 0,43 & 1,0 \\
\hline & $20-40$ & 483 & 401 & 116 & 9 & 5 & 1,1 & 0,22 & 0,9 \\
\hline \multirow{2}{*}{ PVd1 } & $0-20$ & 311 & 411 & 278 & 23 & 23 & 2,8 & 0,12 & 5,6 \\
\hline & $20-40$ & 310 & 370 & 320 & 21 & 23 & 2,3 & 0,10 & 5,4 \\
\hline \multirow{2}{*}{ PVd2 } & $0-20$ & 549 & 310 & 141 & 27 & 10 & 1,8 & 0,18 & 2,2 \\
\hline & $20-40$ & 528 & 296 & 176 & 22 & 11 & 1,5 & 0,14 & 2,4 \\
\hline \multirow{2}{*}{ PVd3 } & $0-20$ & 565 & 336 & 99 & 24 & 7 & 1,3 & 0,19 & 1,0 \\
\hline & $20-40$ & 550 & 342 & 108 & 19 & 7 & 1,3 & 0,19 & 1,0 \\
\hline \multirow{2}{*}{ SGe } & $0-20$ & 522 & 359 & 119 & 21 & 3 & 1,5 & 0,50 & 1,1 \\
\hline & $20-40$ & 519 & 341 & 140 & 14 & 3 & 1,4 & 0,47 & 1,2 \\
\hline \multirow{2}{*}{ SXe } & $0-20$ & 377 & 475 & 148 & 29 & 5 & 3,4 & 0,68 & 1,1 \\
\hline & $20-40$ & 370 & 463 & 167 & 22 & 5 & 2,9 & 0,58 & 1,2 \\
\hline \multirow{2}{*}{ LVd1 } & $0-20$ & 764 & 95 & 141 & 11 & 19 & 1,0 & 0,05 & 4,3 \\
\hline & $20-40$ & 723 & 98 & 179 & 12 & 22 & 1,1 & 0,05 & 4,6 \\
\hline \multirow{2}{*}{ LVd2 } & $0-20$ & 470 & 159 & 371 & 25 & 30 & 2,4 & 0,08 & 4,0 \\
\hline & $20-40$ & 437 & 153 & 410 & 20 & 36 & 2,3 & 0,06 & 4,0 \\
\hline \multirow{2}{*}{ LVdf1 } & $0-20$ & 109 & 362 & 529 & 46 & 103 & 6,7 & 0,07 & 3,7 \\
\hline & $20-40$ & 55 & 318 & 627 & 39 & 100 & 5,5 & 0,06 & 4,3 \\
\hline \multirow{2}{*}{ LVdf2 } & $0-20$ & 61 & 243 & 696 & 21 & 107 & 4,3 & 0,04 & 8,0 \\
\hline & $20-40$ & 50 & 229 & 721 & 17 & 109 & 3,4 & 0,03 & 8,4 \\
\hline \multirow{2}{*}{$\mathrm{LBa}$} & $0-20$ & 126 & 344 & 530 & 41 & 108 & 3,3 & 0,03 & 1,1 \\
\hline & $20-40$ & 68 & 269 & 663 & 39 & 115 & 3,3 & 0,03 & 0,9 \\
\hline \multirow{2}{*}{ VEo } & $0-20$ & 172 & 307 & 521 & 58 & 23 & 7,7 & 0,33 & 0,8 \\
\hline & $20-40$ & 169 & 204 & 627 & 38 & 21 & 7,7 & 0,37 & 0,7 \\
\hline
\end{tabular}


originados de basalto (LVd2, LVdf1, LVdf2, LBa e VEo) apresentam textura argilosa, enquanto os solos oriundos de rochas sedimentares (APt, LVd1, PVd1, PVd3, SGe e SXe) e granitos (PVd2) possuem textura franca a franco-arenosa. A variabilidade nos teores de Fed e Feo está associada ao material de origem dos solos, sendo que os solos originados de rochas sedimentares e granitos apresentam teores de ferro mais baixos em relação aos solos originados de basalto, concordando com os dados obtidos por DALMOLIN et al. (2005).

Os teores de matéria orgânica são considerados médios a baixos, exceto para os solos LVdf1, LBa e VEo, cujos teores variaram entre 38 e $58 \mathrm{~g}$ $\mathrm{kg}^{-1}$. Os dois primeiros situam-se em regiões de altitude mais elevada, onde a matéria orgânica é preservada no solo em função das condições climáticas mais frias e úmidas (KÄMPF \& SCHWERTAMNN, 1983ab; DALMOLIN et al. 2005). No solo VEo, os teores mais elevados de matéria orgânica estão associados às condições de drenagem imperfeita e à interação da matéria orgânica com os argilominerais esmectíticos
(KÄMPF et al., 1995). Os índices de vermelho obtidos da notação Munsell variaram de 0,8 a 8,4. Os solos com matizes entre 2,5YR e 5YR e com cromas mais puros (maiores que 3) apresentaram os maiores índices de vermelho. Estes índices foram baixos para os solos com matizes próximos a $10 \mathrm{YR}$ e cromas $\leq 3$. Os índices de vermelho são maiores conforme aumentam os teores de hematita no solo (KAMPF \& SCHWERTMANN, 1983ab; BARRÓN \& TORRENT, 1986).

Os valores médios dos componentes da cor obtidos por comparação visual e por colorimetria encontram-se na tabela 2. As diferenças nas medidas da cor devem-se principalmente a dois fatores, confirmados por POST et al. (1993) e TORRENT \& BARRÓN (1993): a diferença na sensibilidade espectral entre o olho humano e o colorímetro e a diferença na fonte de iluminação. A sensibilidade espectral é padronizada pelo colorímetro e, no caso dos observadores, varia de um indivíduo para outro. Além disso, o colorímetro, que simula a luz natural, possui um fator de iluminação constante.

Tabela 2 - Comparação das medidas médias de cores pelo método de observação visual com a carta de Munsell e por colorimetria para os diferentes solos analisados.

\begin{tabular}{|c|c|c|c|c|c|c|c|}
\hline \multirow{2}{*}{ Solos } & \multirow{2}{*}{ Profund.(cm) } & \multicolumn{3}{|c|}{ Munsell } & \multicolumn{3}{|c|}{ Colorimetria } \\
\hline & & Matiz* & Valor & Croma & Matiz & Valor & Croma \\
\hline \multirow{2}{*}{$\mathrm{APt}$} & $0-20$ & 10YR $(5,0)$ & 5 & 3 & $8,20 Y R(4,3)$ & 4,44 & 2,37 \\
\hline & $20-40$ & 10YR $(5,0)$ & 6 & 3 & 8,46 YR $(4,4)$ & 4,76 & 2,69 \\
\hline \multirow{2}{*}{ LBa } & $0-20$ & $8,75 Y R(4,5)$ & 4 & 4 & $8,83 Y R(4,5)$ & 3,78 & 3,45 \\
\hline & $20-40$ & 8,5YR $(4,4)$ & 4 & 4 & $8,97 Y R(4,6)$ & 3,67 & 3,24 \\
\hline \multirow{2}{*}{ LVd1 } & $0-20$ & 5,75YR $(3,3)$ & 4 & 6 & 5,73YR $(3,3)$ & 3,97 & 4,04 \\
\hline & $20-40$ & 5,5YR $(3,2)$ & 4 & 5 & 5,63YR $(3,2)$ & 3,74 & 4,09 \\
\hline \multirow{2}{*}{$\mathrm{LVd} 2$} & $0-20$ & 5YR $(3,0)$ & 4 & 4 & 6,12 YR $(3,4)$ & 3,26 & 3,37 \\
\hline & $20-40$ & 5YR $(3,0)$ & 4 & 4 & 6,12 YR $(3,4)$ & 3,31 & 3,42 \\
\hline \multirow{2}{*}{ LVdf1 } & $0-20$ & $5,25 Y R(3,1)$ & 4 & 6 & 6,69YR $(3,7)$ & 3,67 & 4,09 \\
\hline & $20-40$ & $5,25 Y R(3,1)$ & 4 & 6 & 6,37YR $(3,6)$ & 3,67 & 4,39 \\
\hline \multirow{2}{*}{ LVdf2 } & $0-20$ & 3,5YR $(2,4)$ & 4 & 6 & 4,26YR $(2,7)$ & 3,23 & 4,50 \\
\hline & $20-40$ & 3,5YR $(2,4)$ & 3 & 5 & 4,26YR $(2,7)$ & 3,10 & 4,53 \\
\hline \multirow{2}{*}{ PVd1 } & $0-20$ & 4,75YR $(2,9)$ & 4 & 7 & 5,24YR $(3,1)$ & 3,65 & 4,28 \\
\hline & $20-40$ & 4,75YR $(2,9)$ & 4 & 6 & 5,37YR $(3,2)$ & 3,58 & 4,20 \\
\hline \multirow{2}{*}{ PVd2 } & $0-20$ & $8,25 Y R(4,3)$ & 5 & 5 & 7,31YR $(3,9)$ & 4,63 & 3,87 \\
\hline & $20-40$ & $8,5 Y R(4,4)$ & 5 & 5 & 7,26 YR $(3,9)$ & 4,45 & 3,82 \\
\hline \multirow{2}{*}{ PVd3 } & $0-20$ & 9,75YR $(4,9)$ & 5 & 3 & 8,42 YR $(4,4)$ & 4,50 & 2,92 \\
\hline & $20-40$ & 9,75YR $(4,9)$ & 5 & 3 & 8,44 YR $(4,4)$ & 4,46 & 2,99 \\
\hline \multirow{2}{*}{ SGe } & $0-20$ & 10YR $(5,0)$ & 5 & 3 & 7,84 YR $(4,2)$ & 5,01 & 2,57 \\
\hline & $20-40$ & 10YR $(5,0)$ & 5 & 3 & 7,72YR $(4,1)$ & 4,74 & 2,56 \\
\hline \multirow{2}{*}{ SXe } & $0-20$ & 9,75YR $(4,9)$ & 6 & 3 & 8,11 YR $(4,2)$ & 4,73 & 2,73 \\
\hline & $20-40$ & 9,75YR $(4,9)$ & 5 & 3 & 7,98YR $(4,2)$ & 4,45 & 2,54 \\
\hline \multirow{2}{*}{ VEo } & $0-20$ & 9,50 YR $(4,8)$ & 4 & 2 & 8,40 YR $(4,4)$ & 3,65 & 1,74 \\
\hline & $20-40$ & 9,50 YR $(4,8)$ & 4 & 2 & 8,59YR $(4,4)$ & 3,62 & 1,70 \\
\hline
\end{tabular}

* Em parênteses, o valor correspondente à escala proposta por POST et al. (1993). 
Os valores dos coeficientes de correlação entre as medidas de cor realizadas com a carta de Munsell e o colorímetro foram de 0,93, 0,97 e 0,94 para os componentes matiz, valor e croma, respectivamente, indicando haver coerência entre os dois métodos, evidenciando o potencial do equipamento e a habilidade dos observadores para medir a cor do solo. Estas constatações estão de acordo com o trabalho de POST et al. (1993), os quais também encontraram correlação significativa entre as medidas do colorímetro e observações múltiplas com a carta de Munsell. A determinação da cor do solo pelo método visual ocorreu em condições controladas no laboratório, o que permitiu minimizar as diferenças atribuídas ao fator iluminação. Sendo assim, as divergências entre os observadores devem-se principalmente a fatores psicofísicos individuais (BARRÓN et al., 2000). Nos trabalhos de campo, as diferenças podem ser acentuadas devido à não padronização da iluminação.

A análise de variabilidade dos dados da cor obtidos pelos observadores mostrou um elevado coeficiente de correlação: 0,95, 0,95 e 0,93 para matiz, valor e croma, respectivamente. $\mathrm{O}$ matiz apresentou o menor coeficiente de variação, assim como a menor amplitude, com valores maiores apenas para os solos PVd2 e VEo, que apresentaram variação em até 5 pontos (matizes entre 5YR e 10YR). O valor apresentou variabilidade intermediária, com amplitude média de 1,5 pontos e um coeficiente de variação de 12,2\%. Quanto ao croma, o valor médio para o coeficiente de variação foi de 18,5\% e uma amplitude de 2,3 pontos na carta de Munsell, o que significa dizer que houve maior dificuldade dos observadores em avaliar este componente da cor.

Para estudar a correlação entre a matéria orgânica e a cor, os solos foram separados em dois grupos: os solos hematíticos e os demais solos. Os solos hematíticos (LVd1, LVd2, LVdf1 e PVd1) e com matizes mais vermelhos que 6,25YR apresentaram comportamento diferenciado e variação nos teores de matéria orgânica de 17 a $25 \mathrm{~g} \mathrm{~kg}^{-1}$, considerados baixos a médios, sendo que os teores de Fed estão entre 19 e $109 \mathrm{~g} \mathrm{~kg}^{-1}$ (Tabela 1). Foi confirmado que a matéria orgânica teve pouca influência na pigmentação de solos avermelhados, confirmada pela correlação nãosignificativa entre o valor Munsell e o teor de matéria orgânica. Para os demais solos, as correlações entre os teores de matéria orgânica e o valor Munsell determinado por colorimetria foram significantes, com decréscimo do valor conforme o incremento da matéria orgânica (Figura 1a), concordando com SCHULZE et al. (1993), que encontraram correlações significativas entre o valor Munsell e a matéria orgânica, não observando correlação entre o croma e a matéria orgânica.
Os índices de vermelho apresentaram correlações elevadas com o matiz, indicando que a hematita é responsável pela cor avermelhada e confirmando dados da literatura, sendo que o croma e o valor tiveram correlações menores, porém significativas (Figura 1d, 1e, 1f). O Fed, atribuído à presença de óxidos de ferro, apresentou comportamento semelhante, com correlações elevadas e negativas com o valor, principalmente ao separar os solos LBa, LVdf1 e LVdf2 (Figura 1b), que apresentam teores de Fed muito elevados se comparados aos demais solos (Tabela 1), não sendo observadas correlações significativas para o componente matiz. Os solos com maiores índices de vermelho são o LVd1, LVd2, LVdf2 e PVd1. CAMPOS et al. (2003) calcularam os índices de vermelho por meio das coordenadas L*a*b* e da notação Munsell para semiquantificar os teores de hematita, obtendo correlações significativas $(r>0,76)$.

A granulometria apresentou correlações significativas com o componente valor. A areia apresentou as melhores correlações ao separar os solos LVd1 e LVd2, com textura média a arenosa (teores de areia variáveis entre 437 e $764 \mathrm{~g} \mathrm{~kg}^{-1}$ ) e teores médios a baixos de óxidos de ferro. Os solos LVd1 e LVd2 são avermelhados, com matiz na faixa de 5YR, devido ao poder pigmentante da hematita, mesmo em pequenas quantidades (RESENDE et al., 2002). O aumento da proporção da fração areia tende a proporcionar tonalidades (valor Munsell) mais claras aos solos. Este comportamento é influenciado pela presença de minerais claros (quartzo) e por menores teores de matéria orgânica e óxidos de ferro. Para a fração argila, o efeito foi inverso (Figura 1c).

\section{CONCLUSÕES}

Na determinação da cor do solo por meio do uso do colorímetro, foi obtida maior precisão quando comparada à carta de Munsell, por medir em condições controladas e perceber diferenças de cor em pequenos intervalos de matizes, menores que os encontrados entre duas páginas consecutivas da carta de Munsell.

Quanto à determinação de cor de amostras de solo por diferentes observadores, o componente matiz apresenta maior concordância em termos de precisão, seguido pelo valor e, em último lugar, pelo croma.

Os componentes da cor determinados por colorimetria apresentaram correlações significativas com os atributos dos solos analisados (matéria orgânica, índice de vermelho, teores de ferro e granulometria), mostrando que esta é uma técnica 

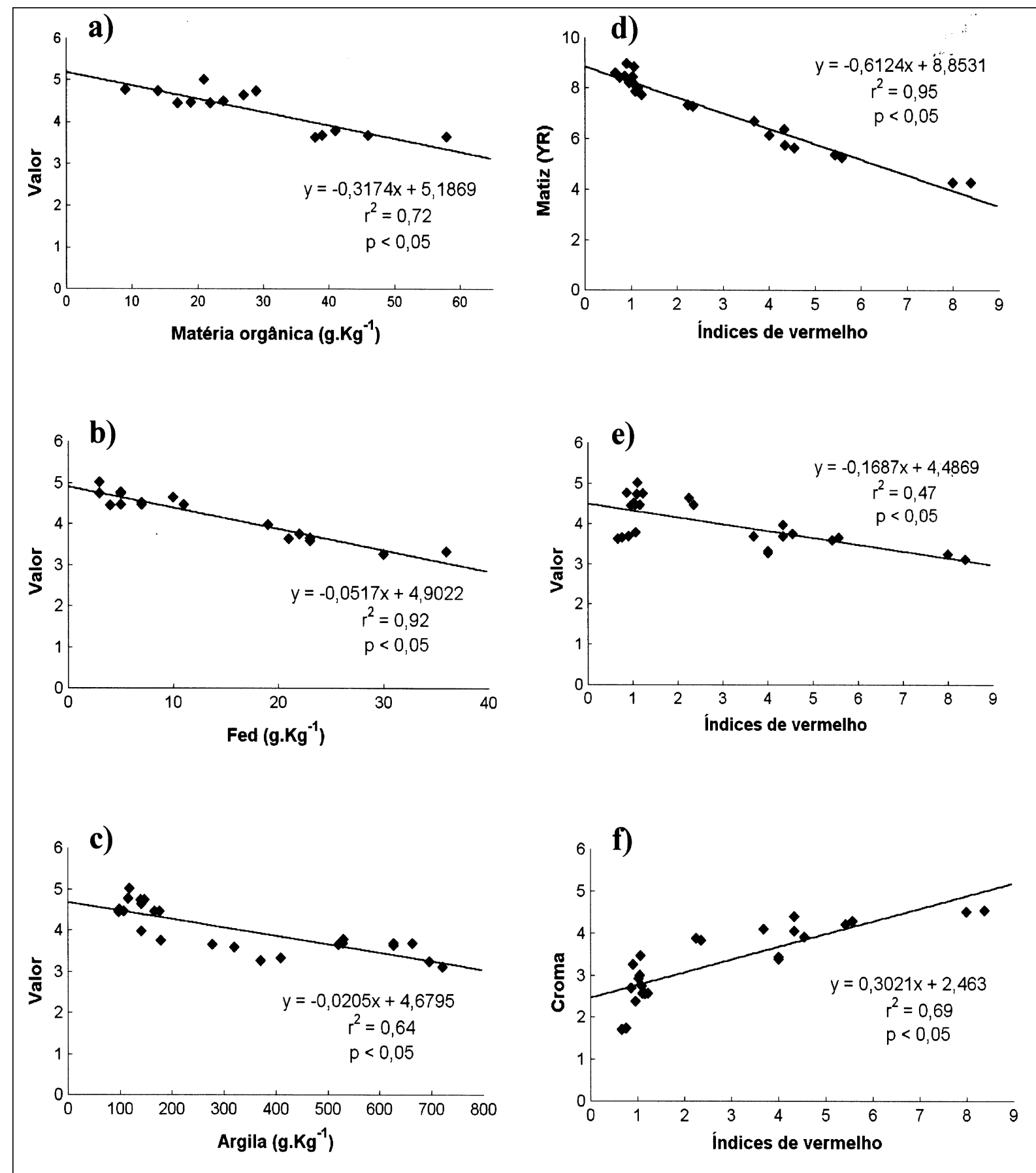

Figura 1 - Relação entre os componentes da cor e os teores de matéria orgânica (a), ferro extraído com DCB - Fed (b), argila (c) e índices de vermelho (d, e, f).

eficiente e promissora para obter informações do solo de maneira simples e de baixo custo.

\section{REFERÊNCIAS}

AZEVEDO, A.C.; DALMOLIN, R.S.D. Solos e ambiente: uma introdução. Santa Maria: Palotti, 2004. 100p.
BARRON, V.; TORRENT, J. Use of the Kubelka-Munk theory to study the influence of iron oxides on soil colour. Journal of soil Science, Oxford, v.37, p.499-510, 1986.

BARRÓN, V. et al. Caracterização de óxidos de ferro em solos por espectroscopia de reflectância difusa. In: NOVAIS, R.F. et al. Tópicos em ciência do solo. Viçosa: Sociedade Brasileira de Ciência do Solo, 2000. V.1, p.139-161.

Ciência Rural, v.36, n.4, jul-ago, 2006. 
CAMPOS, R.C. et al. Determinação indireta do teor de hematita no solo a partir de dados de radiometria e colorimetria. Pesquisa Agropecuária Brasileira. Brasília, v.38, n.4, p.521528, 2003.

CIE. Commision Internationale L'Eclarirage. Proceedings of the eight session. Cambridge: England, 1931. np. (Bureal central de la CIE, Paris).

DALMOLIN, R.S.D. et al. Organic matter characteristics and distribution in ferralsols profiles of a climosequence in southern Brazil. European Journal of Soil Science. 2005. (no prelo)

EMBRAPA. Centro Nacional de Pesquisa de Solos. Sistema brasileiro de classificação de solos. Brasília: EMBRAPA, 1999. 412p. il. (EMBRAPA/CNPS - RJ. Documentos, 5).

EMBRAPA. Manual de métodos de análise de solo. 2.ed. Rio de Janeiro: EMBRAPA, 1997. 212p. il. (EMBRAPA/ CNPS - RJ. Documentos, 1).

HOLMGREN, G.G.S. A rapid citrate-dithionite extractable iron procedure. Soil Science Society of America Journal, v.31, p.210-211, 1967

HUNTERLAB. CIE L*a*b* color scale: applications note, v.8, n.7, 1996. Acesso em 12 de setembro de 2004. On line. Disponível na internet: http://www.hunterlab.com/ color_theory.php.

HURST, V.J. Visual estimation of iron in saprolite. Geoloic Society American, Bull, v.88, p.174-176, 1977.

KÄMPF, N.; SCHWERTMANN, U. Relações entre óxidos de ferro e a cor em solos cauliníticos do Rio Grande do Sul. Revista Brasileira de Ciência do Solo, Campinas, v.7, p.27-31, 1983a.

KAMPF, N.; SCHWERTMANN, U. Goethite and hematite in a climossequence in southern Brazil and their application in classification of kaolinitic soils. Geoderma, Amsterdam, v.29, p.27-39, 1983b.

KÄMPF, N. et al. Alterações mineralógicas em seqüência Vertissolo-Litossolo na região da Campanha no Rio Grande do Sul. Revista Brasileira de Ciência do Solo, Campinas, v.19, p. 349-357, 1995.

MELVILLE M.D.; ATKINSON, G. Soil color: its measurement and its designation in models of uniform color space. Journal of Soil Science, Ottawa, v.36, p.495-512, 1985.
MINOLTA. Precise color communication: color control from perception to instrumentation. Japan, 1998. 59p.

NELSON, D.W.; SOMMERS, L.E. Total carbon, organic carbon and organic matter. In: SPARKS, D.L. et al. (Eds). Methods of soil analysis: chemical methods. Part 3. Madison: American Society of America, 1996. p.961-1010.

POST, D.F. et al. Correlations between field and laboratory measurements of soil color. In: BIGHAM, J.M.; CIOLKOSZ, E.J. Soil color. Madison: Soil Science Society of America, 1993. p.35-49. (Special Publication, 31).

RESENDE, M. et al. Pedologia: base para distinção de ambientes. 4.ed. Viçosa: NEPUT, 2002. 338p.

SCHEINOST, A.C.; SCHWERTMANN, U. Color identification of iron oxides and hydroxysulfates: use and limitations. Soil Science Society of America Journal. Madison, v.63, p.14631471, 1999.

SCHULZE, D.G. et al. Significance of organic matter in determining soil colors. In: BIGHAM, J.M.; CIOLKOSZ, E.J. Soil color. Madison: Soil Science Society of America, 1993. p.71-90. (Special Publication, 31).

SCHWERTMANN, U. Differenzierung der eisen oxide des bodens durch extraktion unit saurer ammoniumoxalat-losung. Zeitschrift für Pflanzenernährung und Bodenkunde, Weinheim, v.105, p.194-202, 1964.

STAT SOFT, Inc. Statistica (Data Analysis Software System), version 6. Tulsa, 2001. Acesso em: 01/09/2005. On line. Disponível em www.statsoft.com.

TORRENT, J.; BARRÓN, V. Laboratory measurement of soil color: theory and pratice. In: BIGHAM, J.M.; CIOLKOSZ, E.J. Soil color. Madison: Soil Science Society of America, 1993. p.21-33. (Special Publication, 31).

TORRENT, J. et al. Iron oxide mineralogy of some soils of two river terrace sequences in Spain. Geoderma, v.23, p.191208, 1980.

YEOMANS, J.C.; BREMNER, J.M. A rapid and precise method for routine determination of organic carbon in soil. Soil Science Plant Analysis, v.19, p.1467-1476, 1988. 